\title{
PEDUNCULAR HALLUCINOSIS AS THE PRESENTING FEATURE OF MIDBRAIN/PONTINE INFARCT
}

F. Islam¹, N. Hannon ${ }^{1}$, K. Khadjooi ${ }^{1}$.

${ }^{1}$ Cambridge University Hospitals NHS Trust, Department of Stroke Medicine, Cambridge, United Kingdom.

\section{Background and Aims}

Visual hallucinations, a common presentation in acute medical patients with infection and psychosis, is a relatively rare presentation of hyperacute stroke. We describe a case of visual hallucinations as the presenting feature of a pontine/midbrain infarct.

\section{Case Report}

An 84-year old female presented with 3-day history of horizontal diplopia and unsteadiness. Past medical history included hypertension, an old lacunar infarct and surgically-corrected bilateral cataract. Onset of symptoms was preceded by short-lived episodes of vivid visual hallucinations whenever she closed her eyes of people with "weird" faces and animals on either side of her visual field approaching her. The hallucinations resolved upon falling asleep and she was able to distinguish them from reality. Sleep was unimpaired.

On examination, right internuclear opthalmoplegia, mild left-sided ataxia and truncal ataxia was found. Visual fields, visual acuity and cognition were normal.

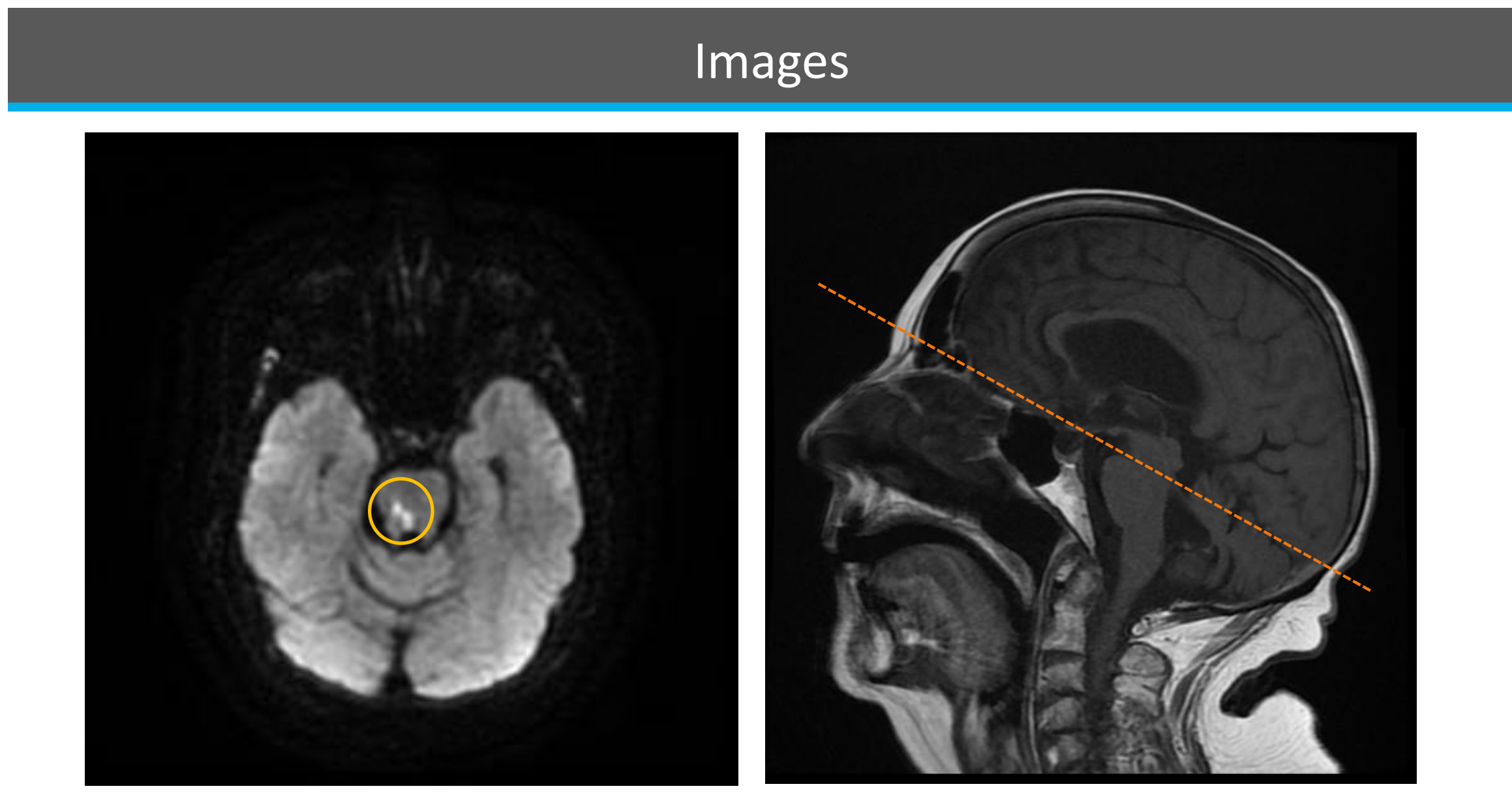

Left: Diffusion-weighted MRI showing area of infarct at the junction of pons and midbrain. Right: T1 weighted MRI in sagittal view, dashed line corresponds to the level at which the image on the left was taken.

\section{Results}

MRI showed an acute infarct at the junction of pons/lower midbrain. She was found to have polycythemia vera, being treated with intermittent venesection. On discharge at day 12, diplopia had resolved.

\section{Conclusion}

Peduncular hallucinosis $(\mathrm{PH})$, a rare form of visual hallucinations, is characterised by acute onset of transient vivid, colourful hallucinations involving moving characters or animals, which are distinguishable from reality. $\mathrm{PH}$ has been reported in midbrain, pontine and thalamic lesions of inflammatory, malignant or most commonly, vascular origin. The likely mechanism is disruption of visual cortex connectivity via damage to thalamocortical pathways.

This case serves as a reminder to consider mesencephalic infarct or haemorrhage in cases of acute onset vivid hallucinations, and to carefully examine for oculomotor disturbances and ataxia.

\section{References}

Benke T. Peduncular hallucinosis: a syndrome of impaired reality monitoring. J Neurol. 2006 Dec; 253(12):1561-1571. Epub 2006 Sep 27

Geddes M, Tie, Gabrieli J, McGinnis S, Golby A, Whitfield-Gabrieli S. Functional Network Reorganization Following Subcortical Stroke: Applying a LesionalFunctional Imaging Approach. Neurology 2016 Apr, 86 (16 Supplement) P4.041

Kölmel HW. Peduncular hallucinations. J Neurol. 1991 Dec; 238(8):457-459

Manford M, Andermann F. Complex visual hallucinations. Clinical and neurobiological insights. Brain. 1998 Oct; 121((Pt 10)):1819-1840.

Notas K, Tegos T, Orologas A. A case of peduncular hallucinosis due to a pontine infarction: a rare complication of coronary angiography. Hippokratia. 2015 Jul-Sep; 19(3): 268-269. 